\title{
A Prospective Comparative Study between the Software Models and Clinical Models of Clear Aligner Treatment
}

\author{
Dr Arisha Izhar,' Dr Gurkeerat Singh,2 Dr Varun Goyal,3 Dr Rajkumar Singh," \\ Dr. Nishant Gupta, 5 Dr. Prerna Pahuja ${ }^{6}$ \\ 'Senior Lecturer, ${ }^{2}$ Professor and Head, ${ }^{3.45}$ Associate Professor, ${ }^{6}$ Post Graduate Student, Department of Orthodontics, Srcdsr, Faridabad
}

Correspondence: Dr Arisha Izhar; Email: arishaizhar@gmail.com

\section{ABSTRACT}

Introduction: The purpose of this prospective clinical study was to compare the three dimensional predicted software models with the stage clinical STL models and to evaluate the efficacy of tooth movement with clear aligners.

Materials \& Method: The sample size included 10 cases with mild anterior crowding treated with aligner therapy. The virtual model of the predicted tooth position was superimposed on the virtual model of the achieved tooth position at various stages over their stationary posterior teeth by using MeshLab software. The amount of tooth movement predicted was compared with the amount of tooth movement achieved.

Result: The results of this study have shown that when a comparison was made on the basis of irregularity scores in both the groups, it was seen that the irregularity score was more in Clinical STL group at each stage such as 2.55 at T4, 1.65 at T6 and 1.0 at T8 whereas 2.0 at T4. 0.90 at T6 and 0.25 at T8 in the Software model group. Also, On comparing mean accuracy these three stages, the analysis of data showed the mean accuracy at T4 is $62.5 \%$, mean accuracy at T6 is $68.8 \%$ and the mean accuracy at T8 is $78.1 \%$.

Conclusion: The predicted software models do not accurately reflect the patient's tooth position .There is an overestimation by predicted software as compared to actual clinically achieved tooth position. There is a need of overcorrection to be built in the treatment planning stage itself and execution of the anticipated end result.

Keywords: Clear aligner, ClinCheck, Orthodontic tooth movement, Stereolithography

\section{INTRODUCTION}

Movement of teeth without the use of bands, brackets or wires was described as early as 1945 by Dr H. D. Kesling. He reported the use of a flexible tooth positioning appliance. Later, Nahoum ${ }^{2}$ and others wrote about various types of overlay appliances such as invisible retainers.

Minor tooth movements have also been achieved with a technique developed by Raintree Essix (New Orleans, LA). This technique used clear aligners formed on plaster models of the teeth. This type of appliance was effective in correcting mild discrepancies in the alignment of teeth. ${ }^{3-5}$ However, movements are limited to $2-3 \mathrm{~mm}^{4}$ and beyond this range, another impression and a new appliance were advocated.

Today in this modern world of orthodontics, various new techniques have been developed to make the treatment more comfortable and esthetic for the patient. The patient has a plethora of options to choose from based on factors such as cost, treatment time, esthetics, comfort and so on. Owing to these factors, increasing numbers of adult patients have sought orthodontic treatment and demand for aesthetic appliances has increased in recent years. ${ }^{6}$

With further advancement in orthodontic technology, Align Technology introduced Invisalign ${ }^{\mathrm{TM}}$ in 1998, a series of removable polyurethane aligners, as an esthetic alternative to fixed labial appliances. Usually scanned images are converted to physical models by using different stereolithography techniques to fabricate series of aligners that sequentially reposition the teeth. ${ }^{7.8}$ Stereolithographic models are constructed at every stage. ' Each aligner is programmed to move a tooth or a small group of teeth 0.25 to $0.33 \mathrm{~mm}$ every 14 days. ${ }^{10}$

Since there can be many variables that could affect tooth movement, ${ }^{6}$ these variables can be biological factors such as periodontal ligament, age and sex of patient, root length, bone levels, bone density, 
medications, certain systemic conditions can have inhibitory, synergistic, or additive effects on Orthodontic tooth movement(OTM)." Variability among patients can affect OTM. Hence, it is necessary to evaluate the difference between the predicted and actual teeth movement achieved. Consistently performing these analyses during treatments will provide a useful database that could be used to study treatment progress and variables affecting movement over time. There is lack of literature that determines the deviation of the clinical outcome of clear aligners with their predicted outcome. No in Vivo study has compared the predicted and stage clinical treatment outcome. Also no study has been conducted at different stages of aligner therapy to measure the disparity in predicted and achieved outcome. In the fast growing aligner market it is essential to know the efficacy of the appliance being used. Hence there is a need to evaluate and compare the clinical and predicted treatment outcome of clear aligners. The aim of this study is to evaluate predicted treatment outcome of clear aligner, evaluate clinical treatment outcome and to compare the predicted and clinical treatment outcome.

\section{MATERIALS AND METHOD}

Materials used in the study are Vinylpolysiloxane impression material (Putty and light flow), interproximal gauges, interproximal discs, interproximal strips, orthodontic bonding materials Etchants, bonding agent, composite, Impression trays. For scanning and measuring Extra oral dental scanner- Maestro 3D MDS400, Meshlab software (Developed at the Visual Computing Lab at ISTI-CNR with the support of the 3D-co-form project).

The Source of the patients was patients visiting Department of orthodontics and dentofacial orthopaedics, who were indicated for comprehensive orthodontic treatment. ${ }^{10}$ orthodontic patients having mild to moderate crowding in lower incisors were scheduled for regular evaluation using Little's Irregularity Index. ${ }^{12}$ The patients included for this study was adult patients, healthy, compliant and motivated patients who can visit the department regularly. Mild to moderate lower anterior crowding according to Little's irregularity index. Non extraction treatment plan in lower arch. The tray used for treatment should not be altered with scissors or thermopliers. Exclusion was based on severe crowding, large restorations in lower anterior teeth, prosthetic replacements in lower anterior teeth, gross gingival /periodontal problems in lower anterior teeth, recent extraction and tooth trauma

Impressions were taken repeatedly with polyvinylsiloxane at different stages and sent to laboratory for 3D scan of dentition and to make a virtual model of the cast. After completing the initial series of aligners, polyvinyl siloxane impressions was taken, ${ }^{13-14}$ at various stages starting from stages T4,T6 and T8, and mailed to Kline Technology. The clinical models were scanned using the Extra oral dental scanner- Maestro 3D MDS400 (Figure 1) and converted to a stereo lithography (STL) format. An STL file was created for each set of models for maxillary and mandibular arch separately. The software model files were also converted to the STL format. Mesh lab software (Figure 2) with the support of the 3D-co-form project program used to make digital measurements and derive the alignment, irregularity scores on both the models and compare the achieved teeth position at different stages.

The Mesh Lab software allowed the measurements to be made using a measuring tool, a software application. The software enables to reproducibly superimpose 2 digital models on user-selected reference points, such as untreated posterior teeth. The digital models at various stages are superimposed over the untreated stationary premolars and molars. With the help of measuring tool, it measured the resolution of crowding, rotation and alignment of each anterior teeth (Figure 3).

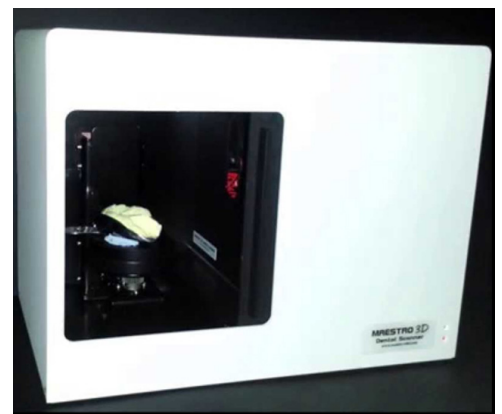

Figure 1: Impression scanning

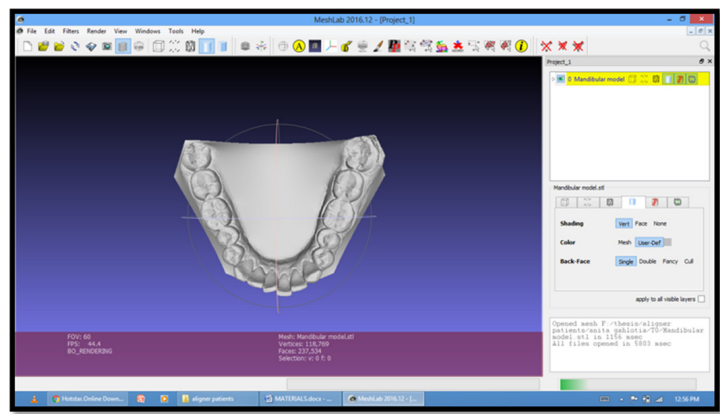

Figure 2: Digital model

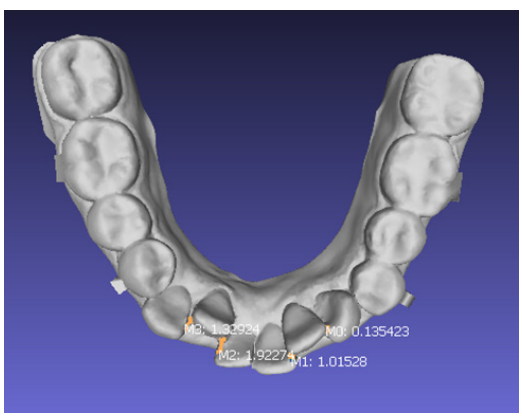

Figure 3: Segmentation 

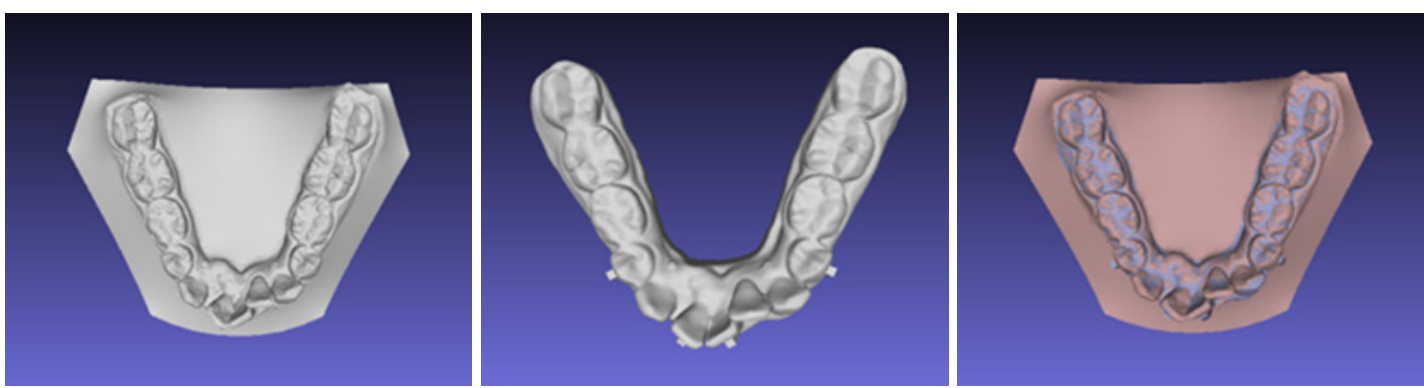

Figure 4: Stage 1
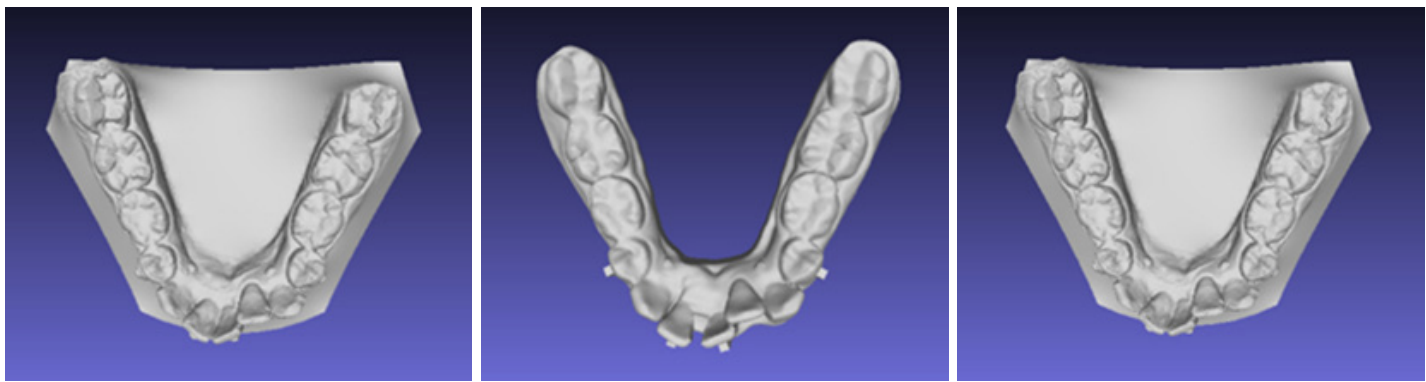

Figure 5: Stage 2
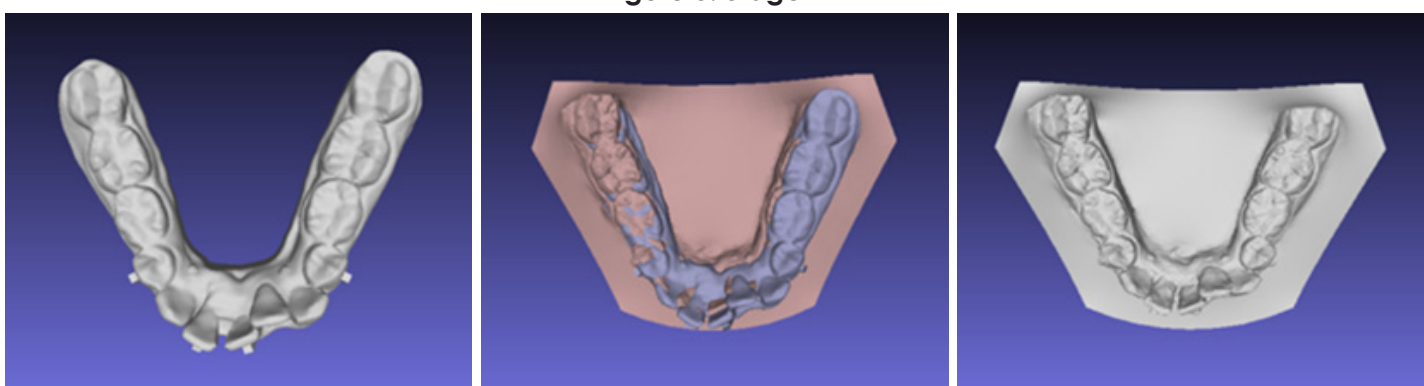

Figure 6: Stage 3
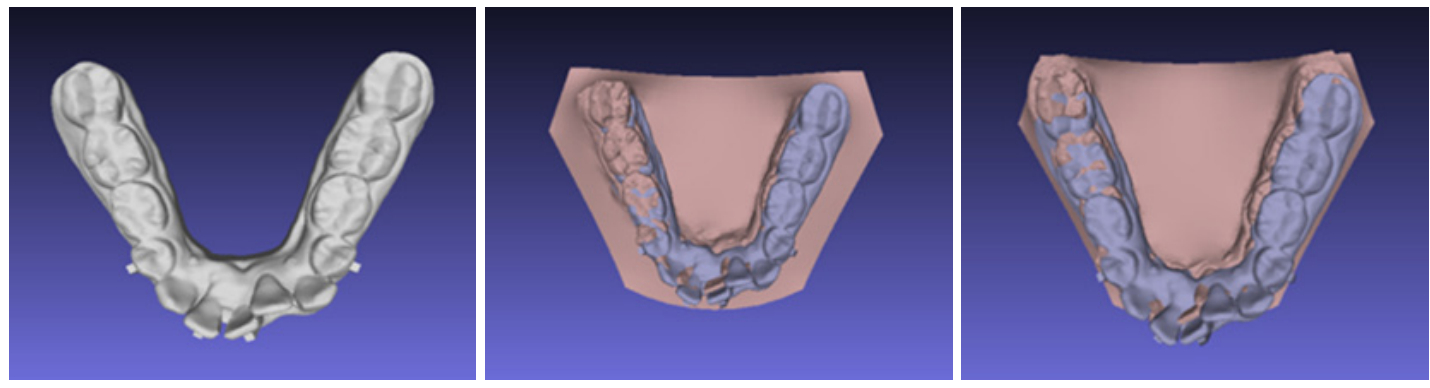

Figure 7: Stage 4

The difference between the scores for the clinical model and the software model is calculated for the total score and /or discrepancy.

The Clinical and software STL models of Zero aligner at T0 stage, aligner at T4, aligner at T6, aligner at T8 are taken and superimpositions are done (Figure 4-7).

Once 2 models are superimposed, software will perform an efficacy analysis report which will show quantitative measurements for predicted and achieved movements. The percentage of accurate tooth movement will be determined by the following equation:
Percentage of accuracy $=100 \%$ - [(| predictedachieved | / | predicted | | *100\%]

\section{RESULT}

This study was done to assess the difference between the stage clinical outcome and the predicted outcome of clear aligners and also percentage of accuracy. Data was entered into Microsoft Excel spreadsheet and was checked for any discrepancies. Summarized data was presented using Tables and Graphs. The data was analysed by SPSS (21.0 version) and Epi-info version 3.0. Shapiro Wilk test was used to check which 
Table 1: Comparison between T0 and T4

\begin{tabular}{|l|c|c|c|c|c|}
\hline \multicolumn{1}{|c|}{ Change from T0 to T4 } & Mean & Std. Deviation & Mean difference & t-test value & p-value \\
\hline STL model & 0.70 & 0.26 & -0.55 & -3.498 & 0.007 \\
\hline Software model & 1.25 & 0.42 & & & \\
\hline
\end{tabular}

Table 2: Comparison between TO and T6

\begin{tabular}{|l|c|c|c|c|c|}
\hline \multicolumn{1}{|c|}{ Change from T0 to T6 } & Mean & Std. Deviation & Mean difference & t-test value & p-value \\
\hline STL model & 1.60 & 0.32 & -0.75 & -6.708 & 0.000 \\
\hline Software model & 2.35 & 0.41 & & & \\
\hline
\end{tabular}

Table 3: Comparison between T0 and T8

\begin{tabular}{|l|c|c|c|c|c|}
\hline \multicolumn{1}{|c|}{ Change from T0 to T8 } & Mean & Std. Deviation & Mean difference & t-test value & p-value \\
\hline STL model & 2.25 & 0.35 & -0.75 & -4.392 & $0.002^{*}$ \\
\hline Software model & 3.00 & 0.82 & & & \\
\hline
\end{tabular}

all variables were following normal distribution. Paired or Dependent t-test was used for comparison of 2 mean values obtained from a same group or a pair of values obtained from the same sample when the data follows normal distribution. The $\mathrm{p}$-value was taken significant when less than $0.05(p<0.05)$ and Confidence interval of $95 \%$ was taken

In this study the mean change from TO to T4, T0 to T6 and TO to T8 was compared from Clinical models and Software models and it was seen that the mean change was more in the Software models at each stage respectively. The mean accuracy of the clear aligners was around $78 \%$ at T8.

The mean change from T0 to T4 was compared between Clinical and Software models using the Paired t-test. The mean change from T0 to T4 was significantly more in Software model, that is 1.25in comparison to Clinical model which is 0.70 (Figure 8) (table 1).

The mean change from TO to T6 was compared between STL and Software models using the Paired t-test. The mean change from T0 to T6 was significantly more in Software model that is 2.35 in comparison to Clinical model which is 1.60 (Figure 9)(table 2).

The mean change from T0 to T8 was compared between STL and Software models using the Paired t-test. The mean change from T0 to T8 was significantly more in Software model that is 3.00 in comparison to Clinical model which is 2.25(Figure 10) (table 3).

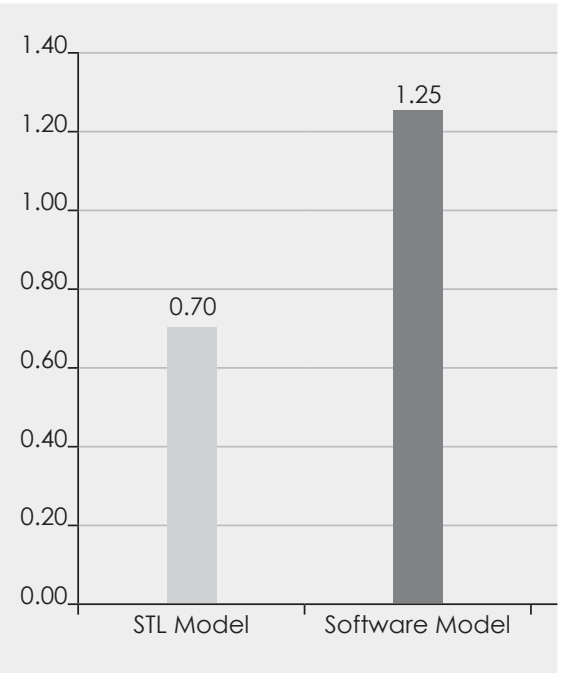

Figure 8: Mean Change from T0 to T4

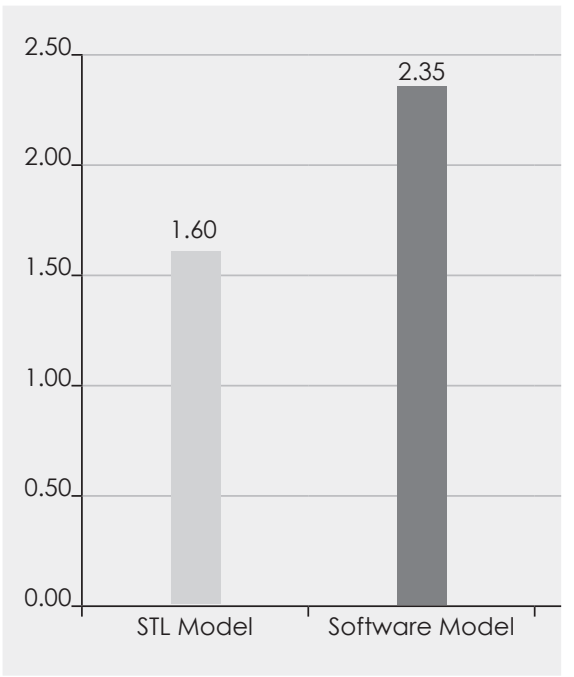

Figure 9: Mean Change from T0 to T6

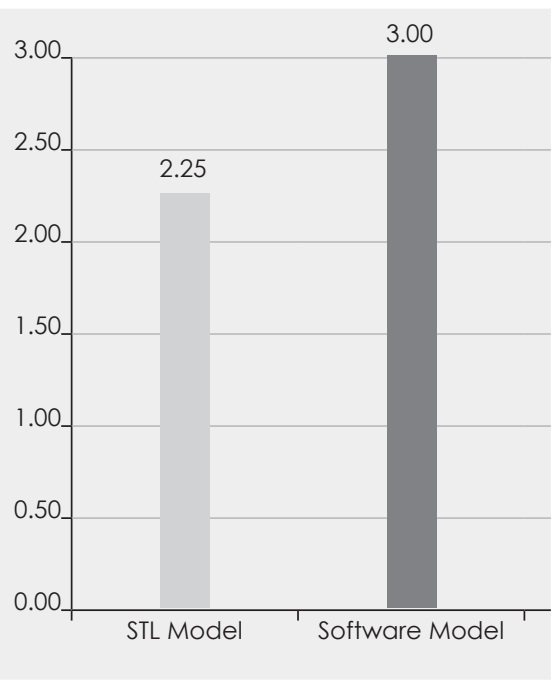

Figure 10: Mean Change from T0 to T8 
Table 4: Accuracy among T4, T6 and T8

\begin{tabular}{|c|c|c|}
\hline Accuracy & Mean & Std. Deviation \\
\hline T4 & $62.50 \%$ & $29.20 \%$ \\
\hline T6 & $68.83 \%$ & $13.05 \%$ \\
\hline T8 & $78.12 \%$ & $13.84 \%$ \\
\hline
\end{tabular}

Table 5: Descriptive statistic between STL model and Software model

\begin{tabular}{|c|c|c|c|c|}
\hline \multirow{2}{*}{} & \multicolumn{2}{|c|}{ STL model } & \multicolumn{2}{c|}{ Software model } \\
\cline { 2 - 5 } & Mean & SD & Mean & SD \\
\hline T0 & 3.25 & 1.16 & 3.25 & 1.16 \\
\hline T4 & 2.55 & 1.26 & 2.00 & 1.11 \\
\hline T6 & 1.65 & 1.16 & 0.90 & 0.99 \\
\hline T8 & 1.00 & 0.91 & 0.25 & 0.42 \\
\hline
\end{tabular}

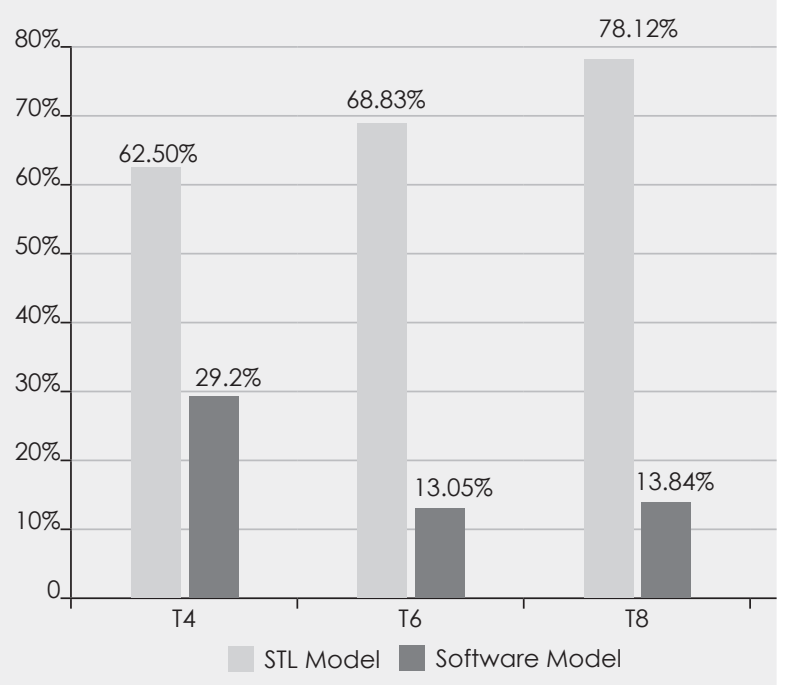

Figure 11: Accuracy

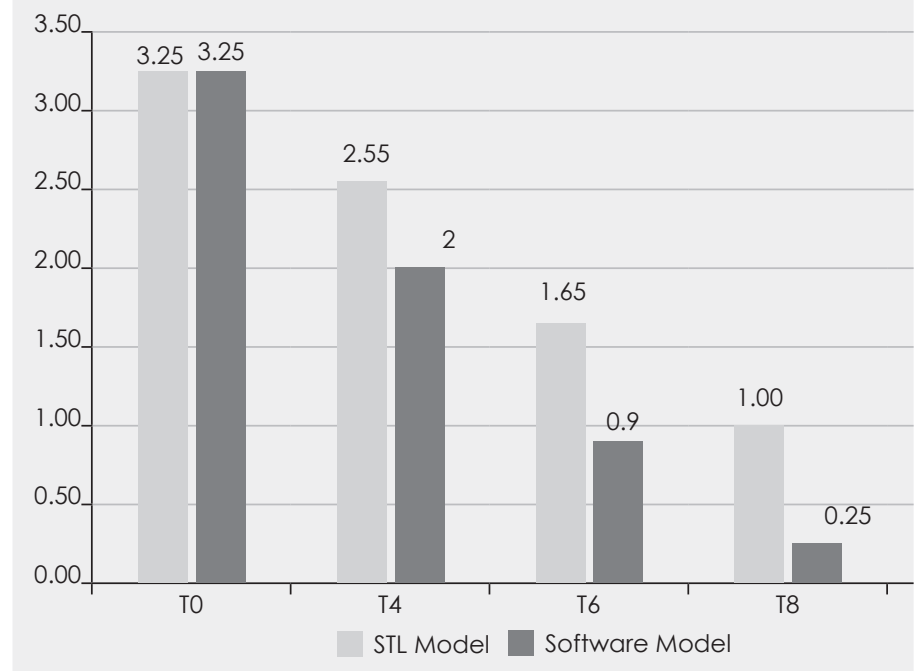

Figure 12: Comparative evaluation

Also, the evaluation of the mean accuracy of clear aligners in clinical models at T4 was found to be 62.5 , 68.83 at T6 and 78.12 at T8 (Figure 11)(table 4).

Moreover, the comparative evaluation of the irregularity score of Clinical and Software models has been depicted at T0 which is 3.25,3.25 respectively, at T4 2.55,2.00 respectively, at T6 $1.60,0.90$ and at T8 stage which is $1.00,0.25$ respectively (Figure 12) (table 5).

\section{DISCUSSION}

In 2005 Lagrave're and Flores-Mir ${ }^{15}$ published a systematic review in which only two studies met their inclusion criteria related to Invisalign ${ }^{\text {TM }}$ therapy efficacy. ${ }^{16,17}$ It was stated that no strong conclusions could be made regarding the treatment effects of

this kind of orthodontic treatment. Thus, clinicians who plan to use Clear Aligner Treatment(CAT) on their patients have to rely on their clinical experience, the opinions of experts, and limited published evidence. The purpose of the present study was to compare a proprietary software model with the actual clinical outcome to determine whether overall occlusion and the crowding at various stages of aligners such as aligner no 4, 6 and 8 is comparable.

The results of this study shows that mean change from TO to T4, TO to T6 and TO to T8 comparing both the groups was significantly more in software models in comparison to clinical models.

The result gave an inference that the clinical models showed resolution of crowding when it is assessed 
individually at different stages. But, when it is compared with the software models at different stages the mean change is lesser in clinical models as compared to software models, which helps us to conclude that resolution of crowding is better in the software models and it overestimates the correction of the crowding and misalignment.

The comparison was made for the mean accuracy of the clear aligners at the different stages of aligners. The analysis of data showed the mean accuracy which concluded from the data that the maximum accuracy matched for both the groups at the T8 stage, though the accuracy of this match was lesser in the initial stages of treatment, the accuracy between the predicted and clinical outcomes improves as the treatment progressed.

Kravitz ${ }^{10}$ et al conducted a prospective clinical study in 2009 to evaluate the efficacy of tooth movement with Invisalign ${ }^{\text {TM }}$. The amount of tooth movement predicted by ClinCheck (Align Technology) was compared with the amount achieved after Invisalign ${ }^{\text {TM }}$ treatment. Tooth movement was evaluated on Tooth-Measure, Invisalign's proprietary virtual model superimposition software. It concluded that the mean accuracy of tooth movement with Invisalign was $41 \%$. 18,19

Also, Buschang ${ }^{7}$ conducted a prospective study which Compared with the patients' models taken immediately after treatment, ClinCheck ${ }^{\mathrm{TM}}$ models overestimated alignment, buccolingual inclinations, occlusal contacts and relations.

Digital computerization allows visualization of the treatment plan not only at beginning and end but also step by step, aligner by aligner throughout the treatment which purportedly reflect the treatment outcomes and hence the anticipated end result can be visualized. But there is no study that correlates and compares the predicted software models and the clinical outcome at varied stages along with the variables in the patients mouth into consideration, as they can alter the clinical outcome end results.

This study was one of a kind where the comparison was made at different stages to assess the efficacy and the accuracy of the aligners and to correlate it with the predicted outcomes. Also, the comparison showed that the accuracy of the appliance is around $78 \%$, which is more than quoted by other other authors in their study.

Also, a study by Drake et al who stated that bodily movement is not achievable by the CAT, ${ }^{20}$ the aligners can easily tip the tooth crown but cannot tip the root because of the inadequate root control movement with aligner system. Although, the tooth movement programmed by the software is bodily movement, tipping of the teeth occurs. And hence, the end result will vary from the programmed or predicted result.

Another study was done by Clements et $\mathrm{al}^{21}$ using Align Technology to compare 2 different materials of the aligner(soft and hard) and. The hard material group showed the best results in PAR score reduction. The stiffness of the material is an important factor in achieving the desired result as it has better tooth control.

These variables along with wear of the aligners by the patient for requisite hour is an important factor in achieving the predicted end result whish should be taken into consideration. Emphasizes should be given to the need of overcorrection to be build in the software, effective attatchment designs so as to make aligners more reliable in terms of treating difficult maloclussions and in order to get the desired result. This study was done using an aligner system with the same propietery software so as to maintain uniformity on all patients and results. However more studies should be done on similar pattern involving more number of patients and also further studies needs to be done to evaluate the expression of the torque with the aligner system and also the material qualities.

\section{CONCLUSION}

The study concluded that the software models overestimated the alignment and the resolution of crowding in comparison with the actual clinical models. There are variables or biological restrains that alter the accuracy of the clear aligner treatment

Hence, there is a need of overcorrection to be built in the treatment planning stage itself and execution of the anticipated end result so as to achieve the desired correction as seen in software models.

\section{OJN}




\section{REFERENCES}

1. Kesling HD. The philosophy of the tooth positioning appliance. Am J Orthod 1945;31:27-304.

2. Nahoum HI. The vacuum formed dental contour appliance. N Y State Dent J 1964;9:385-9

3. Acar YB, Kovan A, Ates M. How Efficient Are Clear Aligners? Clear Aligners Vs Traditional Orthodontic treatment: A Systematic Review. Turkish J Orthod; 2015;27:106-110

4. McNamara JA, Brudon WI. Orthodontics and Dentofacial Orthopedics. Ann Arbor, MI: Needham Press; 2001.

5. Djeu G, Shelton C, Maganzini A. Outcome assessment of Invisalign and traditional orthodontic treatment compared with the American Board of Orthodontics objective grading system. Am J Orthod Dentofacial Orthop. 2005 Sep;128(3):292-8.

6. Melsen B. Northcroft Lecture. How has the spectrum of orthodontics changed over the past decades. Journal of Orthodontics, Vol. $38,2011,134-143$

7. Buschang PH, Ross M, Shaw SG. Predicted and actual end-of-treatment occlusion produced with aligner therapy. Angle Orthod; 2015 85; 723-27

8. Zhang XJ, He L, Guo HM. Integrated three dimensional digital assessment of accuracy of anterior tooth movement using clear aligners: Korean J Orthod;2015;45(6):275-81

9. Boyd RL and Waskalic V. Three-Dimensional Diagnosis and Orthodontic Treatment of Complex Malocclusions With the Invisalign Appliance. Semin Orthod 2001;7:274-293.

10. Kravitz ND, Kusnoto B, BeGole E, Obrez A, Agran B. How well does invisalign work? A prospective clinical study evaluating the efficacy of tooth movement with invisalign. Am J Orthod Dentofacial Orthop 2009; 135:27-35

11. Chisari JR, MCGorray SP, Nair M, Wheelerd TT . Variables affecting orthodontic tooth movement with clear aligners. Am J Orthod Dentofacial Orthop 2014;145:S82

12. Little R M: The irregularity index: a quantitative score of mandibular anterior alignment. American Journal of Orthodontics 1975 Volume 68, Issue 5: 554-563

13. Kuo E, Miller RJ. Automated custom-manufacturing technology in orthodontics. Am J Orthod Dentofacial Orthop 2003;123:578-81

14. Wong BH. Invisalign A to Z. Am J Orthod Dentofacial Orthop 2002;121:540-1

15. Lagrave re MO, Flores-Mir C. The treatment effects of Invisalign orthodontic aligners: a systematic review. J AmDent Assoc. 2005; 136:17241729

16. Rossini G, Parrini S, Castroflorio T, Deregibus A, Debernardi CL Efficacy of clear aligners in controlling orthodontic tooth movement.A systematic review. Angle Orthod. 2015 Sep;85(5):881-9.

17. Align Technology, Inc. The Invisalign Reference Guide. Santa Clara, CA: Align Technology, Inc; 2002

18. Gomez JP, Pena FM, Martinez V, Giraldo DC. Initial force systems during bodily tooth movement with plastic aligners and composite attachments. Angle Orthod. 2015 May;85(3):454-60.

19. Khosravi R, Cohanim B, Hujoel P. Management of overbite with the Invisalign appliance; Am J Orthod Dentofac Orthop; $2017 ; 151: 69$.

20. Drake CT, McGorray SP, Dolce C, Nair M, Wheeler TT. Orthodontic Tooth Movement with Clear Aligners. Interna-tional Scholarly Research Network - ISRN Dentistry Volume 2012, Article ID 657973, 7 pages, doi: 10.5402/2012/657973

21. Clements KM, Bollen AM, Huang G, King G, Hujoel P, Ma T.Activation time and material stiffness of sequential remov-able orthodontic appliances. Part 2: dental improvements. Am J Orthod Dentofacial Orthop. 2003;124:502-508 